\title{
TRANSTORNO MENTAL COMUM ENTRE ACADÊMICAS DE GRADUAÇÃO EM ENFERMAGEM E FATORES ASSOCIADOS
}

\section{COMMON MENTAL DISORDER AMONG NURSING GRADUATION ACADEMICS AND ASSOCIATED FACTORS}

\section{TRASTORNO MENTAL COMÚN ENTRE ESTUDIANTES UNIVERSITARIOS DE ENFERMERÍA Y FACTORES ASOCIADOS}

\author{
Bibiane Dias Miranda ${ }^{1}$, Flávia Buranelo Maito ${ }^{2}$, Maysa Alvarenga Ferreira ${ }^{3}$, Larissa Peres ${ }^{4}$, \\ Mariana Torreglosa Ruiz ${ }^{5}$, Bethania Ferreira Goulart ${ }^{6}$
}

Como citar esse artigo: Miranda BD, Maito FB, Ferreira MA, Peres L, Ruiz MT, Goulart BF. Transtorno mental comum entre acadêmicas de graduação em enfermagem e fatores associados. Rev Enferm Atenção Saúde [Internet]. 2021 [acesso em:__]; 10(3):e202136. doi: https://doi.org/ 10.18554/reas.v10i3.4691

\section{RESUMO}

Objetivo: Identificar a presença de sintomas indicativos de Transtorno Mental Comum, entre acadêmicas do curso de um curso de Graduação em Enfermagem de uma universidade federal e a sua associação com variáveis sociodemográficas, econômicas e comportamentais. Métodos: Trata-se de um estudo observacional e transversal com 163 acadêmicas. Foi utilizado o instrumento Self-Reporting Questionnaire para identificação do Transtorno Mental Comum. Resultados: Entre as participantes a prevalência de Transtorno Mental Comum foi de 44,7\%, associado ao hábito de beber e possuir doença crônica. Conclusão: Os resultados do estudo evidenciaram que é fundamental conhecer as condições de saúde mental de universitários e os fatores que podem estar relacionados para o planejamento de ações específicas e melhor aproveitamento no âmbito acadêmico e na sua qualidade de vida. Descritores: Saúde mental; Saúde da mulher; Enfermagem; Estudantes de enfermagem.

\footnotetext{
1 Doutora. Professora Adjunto do Curso de Graduação em Enfermagem. Universidade Federal do Triângulo Mineiro, UFTM, Uberaba-MG. http://orcid.org/0000-0001-7369-5745

2 Enfermeira. Graduação pela Universidade Federal do Triângulo Mineiro, UFTM, Uberaba-MG. http://orcid.org/0000-0002-5465-1947

3 Doutoranda no Programa de Pós Graduação em Stricto Sensu em Atenção à Saúde. Universidade Federal do Triângulo Mineiro, UFTM, Uberaba-MG. http://orcid.org/0000-0002-4483-0693

4 Enfermeira na Unidade de Atendimento Integrado Pampulha. Belo Horizonte-MG. http://orcid.org/0000-0003$\underline{3169-8133}$

5 Doutora. Professora Adjunto do Curso de Graduação em Enfermagem. Universidade Federal do Triângulo Mineiro, UFTM, Uberaba-MG. http://orcid.org/0000-0002-5199-7328

6 Doutora. Professora Adjunto do Curso de Graduação em Enfermagem. Universidade Federal do Triângulo Mineiro, UFTM, Uberaba-MG. http://orcid.org/0000-0003-2855-6767
} 


\begin{abstract}
Objective: Identify the presence of symptoms indicative of Common Mental Disorder among undergraduate students of a Federal University undergraduate nursing course and its association with sociodemographic, economic and behavioral variables. Method: This is an observational and cross-sectional study with 163 academics. The Self-Reporting Questionnaire instrument was used to identify Common Mental Disorder. Results: Among participants, the prevalence of Common Mental Disorder was $44.7 \%$, associated with drinking and chronic disease. Conclusion: The results of the study showed that it is essential to know the mental health conditions of university students and the factors that may be related to the planning of specific actions and better use in the academic field and in their quality of life.
\end{abstract}

Descriptors: Mental Health; Women's Health; Nursing; Students, Nursing.

\title{
RESUMEN
}

Objetivo: Identificar la presencia de síntomas indicativos de trastorno mental común entre estudiantes de pregrado de un curso de pregrado de enfermería de la Universidad Federal y su asociación con variables sociodemográficas, económicas y de comportamiento. Método: Este es un estudio observacional y transversal con 163 académicos. El instrumento del cuestionario de autoinforme se utilizó para identificar el trastorno mental común. Resultados: Entre los participantes, la prevalencia del trastorno mental común fue del $44,7 \%$, asociada con el consumo de alcohol y las enfermedades crónicas. Conclusión: Los resultados del estudio mostraron que es esencial conocer las condiciones de salud mental de los estudiantes universitarios y los factores que pueden estar relacionados con la planificación de acciones específicas y un mejor uso en el campo académico y en su calidad de vida.

Descriptores: Salud Mental; Salud de la Mujer; Enfermería; Estudiantes de Enfermería.

\section{INTRODUÇÃO}

O Transtorno Mental Comum (TMC) definido por Goldberg e Huxley, por meio de um modelo biossocial, referese a um transtorno mental não psicótico caracterizado por um conjunto de sintomas depressivos, estados de ansiedade, irritabilidade, fadiga, insônia, sonolência, dificuldade de memória e concentração e queixas somáticas como tremores, cefaleia e má digestão. ${ }^{1}$ Os TMC são altamente prevalentes na população e impactam no humor, sentimentos e qualidade de vida dos indivíduos. $^{2}$
A literatura tem revelado um aumento desses transtornos nos últimos anos, especialmente em mulheres, o que vai ao encontro de resultados em estudos brasileiros, com população feminina, em que a prevalência desse distúrbio apresentou taxas de $30,67 \% 33,33,8^{4}$, e $35,7 \%$. $^{5}$

O TMC no gênero feminino ocorre por diversas causas, não apresentando um padrão. Dessa forma, estudo observou o aumento da ocorrência dos transtornos associado à escolaridade e o relacionamento com o companheiro. ${ }^{5}$ Outro, naquelas em união estável, com zero a quatro anos de estudos, que faziam 
uso de medicamento controlado, e que informaram diagnóstico prévio/confirmado pessoal ou na família. ${ }^{3}$

Outro fator predisponente está relacionado às pressões biopsicossociais, como o ingresso no meio acadêmico. Estudos apontaram que a exigência dos alunos na obtenção de um bom desempenho escolar é um fator predisponente ao desenvolvimento de pressões, depressão, podendo levar ao abandono do curso, uma vez que esses alunos não se sentem capacitados o suficiente para serem bons profissionais. $^{6-7}$

Em estudo com acadêmicos do curso de enfermagem, o estresse esteve relacionado ao sexo feminino. ${ }^{8} \mathrm{O}$ TMC também pode estar associado a outras causas como qualidade ruim do sono, provavelmente relacionada à sobrecarga de atividades acadêmicas e ansiedade com provas e trabalhos. ${ }^{9}$

As acadêmicas de graduação têm especificidades que devem ser consideradas, além da questão de gênero - o contexto cultural, fatores socioeconômicos, além da distância da família. Sozinhas, podem encontrar obstáculos difíceis de serem enfrentados, até no que se refere à saúde, inclusive, na saúde mental. Portanto, é fundamental a valorização da saúde mental de acadêmicas de graduação, considerando as diferentes condições de vida, estudo e trabalho em que estão inseridas.

Desta forma, acredita-se que a saúde mental das acadêmicas, especificamente, do curso de graduação em Enfermagem, é essencial para sua qualidade de vida e, consequentemente, para a qualidade de seus estudos e conclusão do seu curso. No entanto, são necessárias pesquisas nacionais que identifiquem o TMC, assim como a sua associação com diferentes fatores. A partir dessa identificação, plano de ações pode ser elaborado e colocado em prática pelas instituições de ensino superior, visando um melhor desempenho acadêmico e qualidade de vida dos universitários.

Este estudo teve como objetivo identificar a presença de sintomas indicativos de transtorno mental comum, entre acadêmicas do curso de Graduação em Enfermagem de uma universidade federal e a sua associação com variáveis sociodemográficas, econômicas e comportamentais.

\section{MÉTODO}

Trata-se de um estudo observacional, exploratório, do tipo transversal, realizado no mês de setembro de 2017, com 163 acadêmicas, maiores de 18 anos, do primeiro ao décimo período do curso de Graduação em Enfermagem de uma universidade federal, no interior do estado de Minas Gerais. 
Os critérios de inclusão do estudo foram participantes maiores de 18 anos e as que aceitaram participar do estudo.

A coleta de dados foi realizada por meio de um instrumento autoaplicável, em sala de aula, durante o início ou término das atividades didáticas, mediante $\mathrm{o}$ consentimento e autorização do docente responsável pela aula. As acadêmicas do curso de Graduação em Enfermagem foram convidadas a participar do estudo e esclarecidas sobre a natureza e os objetivos da pesquisa. Após a aceitação e formalização da anuência por meio do Termo de Consentimento Livre e Esclarecido, em duas vias, foi realizada a distribuição do instrumento.

Foram utilizados dois instrumentos: caracterização sociodemográfica, econômica e comportamental e o SelfReporting Questionnaire (SRQ-20), para avaliar a prevalência de TMC. Todos os instrumentos foram autoaplicáveis.

Para a caracterização sociodemográfica, econômica e comportamental foi elaborado um questionário, com questões estruturadas, com base em experiências prévias dos pesquisadores e na literatura científica. As variáveis foram: idade, procedência, cor, estado marital, trabalho remunerado, valor da renda, número de pessoas residentes no domicílio, realização de atividades físicas, realização de atividades de lazer, doença Rev Enferm Atenção Saúde [Online]. Out/Dez 2021; 10(3):e202136 crônica, uso de bebida alcoólica, hábito de fumar, uso de drogas e filhos vivos.

O SRQ-20 foi utilizado para a identificação do TMC, ele é composto por 20 questões, com duas possibilidades de resposta, sim ou não. O ponto de corte utilizado foi igual ou maior que oito respostas positivas. ${ }^{10}$

Foi realizada a análise estatística por meio do software Statistical Package for Social Science (SPSS) versão 20.0. A análise univariada dos dados foi apresentada na forma de distribuição de frequências absolutas (n) e relativas (\%) para as variáveis qualitativas; e valores de média e mediana (medidas de tendência central), desvios-padrão e valores máximo e mínimo (medidas de variação) para as variáveis quantitativas e na análise bivariada, a existência de associação foi verificada por meio de tabelas de contingência e suas respectivas medidas de associação: Teste Qui-Quadrado de Pearson (X), Razões de prevalência (RP) e Razões de chances de prevalência brutas (RCP). Para todos os testes, foram considerados um intervalo de confiança (IC) de 95,0\% e um nível de significância $\alpha$ de cinco \%.

$\mathrm{O}$ projeto foi encaminhado para o Comitê de Ética em Pesquisa (CEP), sendo aprovado com CAAE 65820717.5.0000.5154 e parecer $n^{\circ}$ 2.140.136 (27 de junho de 2017). 


\section{RESULTADOS}

Participaram do estudo 163 acadêmicas de 18 a 39 anos, com média de idade de 22,14 anos (dp=3,68), $102(63,4 \%)$ residiam em Uberaba e $59(36,6 \%)$ eram procedentes de outras cidades. Quanto à cor, consideraram-se de cor branca 96 $(59,6 \%), 44(27,3 \%)$ de cor parda, 18 $(11,2 \%)$ de cor negra e três $(1,9 \%)$ de cor amarela. Em relação ao estado marital, 81 $(50,3 \%)$ não tinham parceiro (a)/namorado(a), $73 \quad(45,3 \%)$ tinha parceiro(a), mas não viviam junto, e sete $(4,3 \%)$ viviam com o companheiro(a) independente do sexo. No que diz respeito ao trabalho remunerado, $143(88,8 \%)$ não tinham trabalho remunerado, sete $(4,3 \%)$ trabalhavam no mercado formal e $11(6,8 \%)$ no mercado informal. No que se refere ao valor de renda individual, obteve-se um mínimo de zero e um máximo de 4000 reais, média de 367,40 reais $(\mathrm{dp}=558,64)$.

No que tange ao número de pessoas que viviam no domicílio com a participante, o estudo apresentou um mínimo de nenhuma pessoa e um máximo de 20 , com uma média de 3,81 (dp=2,15).

Em relação às atividades físicas e de lazer, 73 (45,3\%) das acadêmicas referiram realizar atividades físicas e $120(74,5 \%)$ alguma atividade de lazer.

Entre as participantes, $142(88,2 \%)$ não possuíam doenças crônicas, entre as que afirmaram possuir, $10(52,6 \%)$ referiam Rev Enferm Atenção Saúde [Online]. Out/Dez 2021; 10(3):e202136 outras doenças, cinco $(26,4 \%)$ depressão, dois $(10,5 \%)$ hipertensão arterial e dois (10,5\%) Diabetes Mellitus.

No que diz respeito ao hábito de fumar, $24(14,9 \%)$ eram tabagistas e 92 $(57,1 \%)$ faziam uso de bebida alcoólica, sendo que $33(35,9 \%)$ bebiam uma ou duas vezes/semana, $26(28,3 \%)$ uma a três vezes/mês, $30(32,6 \%)$ menos de uma vez/mês e três $(3,2 \%)$ todos os dias.

No que se refere ao uso de drogas ilícitas, $12(7,5 \%)$ alegaram fazer uso destas, seis $(50,0 \%)$ usavam menos de uma vez/mês, dois (16,7 \%) todos ou quase todos os dias, dois $(16,7 \%)$ uma ou duas vezes/semana e dois $(16,7 \%)$ uma a três vezes/mês.

Considerando a quantidade de filhos vivos, $14(8,7 \%)$ possuíam filhos e 11 $(6,8 \%)$ responderam que a resposta não se aplicava, pois nunca tiveram relação sexual.

Em relação às respostas das acadêmicas de enfermagem à escala SRQ20, no presente estudo, a prevalência de TMC foi de $44,7 \%$.

$\mathrm{Na}$ análise bivariada, associaram-se ao TMC as variáveis: hábito de beber $(p=0,03)$ e possuir doença crônica $(p=0,01)$, Tabela 1.

A Tabela 1 apresenta a análise bivariada das variáveis sociodemográficas, econômicas e comportamentais relacionadas ao TMC, entre as acadêmicas. 
Tabela 1 - Distribuição das acadêmicas do curso de Graduação em Enfermagem, segundo as variáveis sociodemográficas, econômicas, comportamentais e TMC. Uberaba, MG, Brasil, 2017.

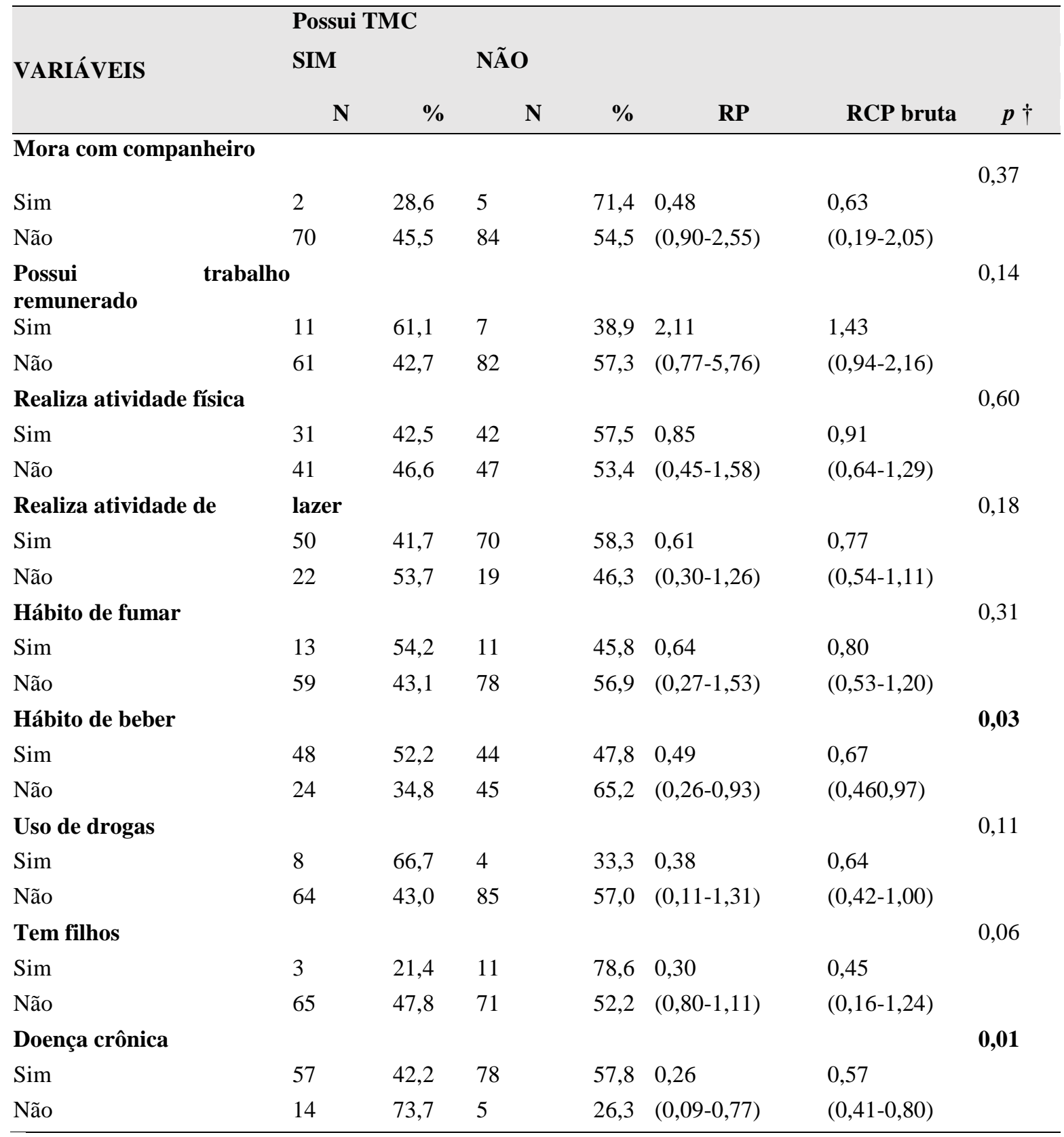

$\dagger$ valor de $p$ para o Teste Qui-Quadrado.

\section{DISCUSSÃO}

A prevalência do TMC, no presente estudo, foi semelhante a resultados de outros estudos, sendo que em algumas pesquisas os resultados foram superiores. Isto foi constatado em um estudo realizado Rev Enferm Atenção Saúde [Online]. Out/Dez 2021; 10(3):e202136 na cidade de Araçatuba-SP, com alunos de graduação em enfermagem, de uma universidade privada, onde a prevalência do TMC foi de $55 \% .^{11}$

Em pesquisa desenvolvida na mesma universidade deste estudo, com 92 
graduandos de enfermagem, o TMC foi de $43,5 \%$ entre os alunos, sendo no sexo masculino $(45,8 \%)$ e entre às mulheres $(43,2 \%)$. Considerando a população feminina, os resultados foram semelhantes; no entanto, ressalta-se que em período de pesquisa diferentes. ${ }^{12}$

Outra pesquisa realizada com 220 estudantes em uma universidade pública do nordeste do Brasil, com estudantes de medicina, odontologia e enfermagem foi evidenciada uma prevalência para o TMC inferior. Destacando-se a prevalência maior nos alunos do curso de enfermagem (39,0\%), seguida do curso de odontologia $(36,8 \%)$ e por último o curso de medicina $(30,1 \%) .{ }^{13}$ Assim como no estudo com universitários de Psicologia e Enfermagem, o TMC foi de $35,71 \%$ entre os estudantes. ${ }^{14}$

A prevalência de TMC identificada, assim como na literatura, é preocupante, visto o impacto que o TMC pode causar na qualidade de vida, no aproveitamento acadêmico e na formação profissional desses acadêmicos.

Em uma pesquisa realizada com alunos de medicina do Sul do Brasil a variável sexo feminino apresentou correlação com o TMC entre os acadêmicos, no final do semestre. ${ }^{9}$

Estudo com estudantes de quatro profissões da área da saúde, na Arábia Saudita, foi encontrada associação entre o sexo feminino e a presença e gravidade da depressão. ${ }^{15}$

No presente estudo, embora os dados foram obtidos apenas com mulheres, identificou-se uma alta prevalência de TMC, como apresentado em estudos comparativos entre os sexos.

Portanto, no que se refere à saúde da mulher, percebe-se a necessidade de mais investigação e ações, visto que as questões específicas que tangem o contexto de gênero podem envolver e estar relacionado à saúde mental e sua relação com a qualidade de vida e futuro profissional.

$\mathrm{Na}$ análise bivariada, o hábito de beber e a presença de doença crônica, entre as acadêmicas, estiveram associados ao TMC.

Em contraste, o uso de bebidas alcoólicas não esteve associado ao TMC em pesquisa desenvolvida com alunos do curso de Graduação em Enfermagem da Universidade Federal do Acre, mesmo que 93,4\% afirmaram fazer consumo de álcool de baixo risco e $6,6 \%$ o uso de risco para o consumo de álcool. ${ }^{16}$

Em relação a possuir doenças crônicas, inquérito domiciliar com 848 mulheres realizado por na cidade de Campinas, SP, de modo semelhante a este estudo identificou que aquelas que relataram várias doenças ou problemas de saúde estiveram mais predispostas a apresentar o TMC. ${ }^{17}$ 
Fatores comportamentais, como o uso de bebidas alcoólicas, devem ser discutidos e analisados em âmbito universitário. A presença de doenças crônicas também merece uma atenção específica em relação ao seu acompanhamento e avaliação. A identificação e suporte em relação a esses fatores podem contribuir para um trabalho voltado para a saúde mental de acadêmicas, como evidenciado nos resultados desse estudo e pesquisa apresentada anteriormente.

Evidenciam-se na literatura nacional, poucos estudos sobre serviços de atendimento psicológicos a estudantes universitários. O que leva a refletir sobre a necessidade, por parte das universidades, de discussão e medidas de atenção e manejo visando o bem-estar dos alunos no ambiente universitário. $^{18}$

Isto pode ser realizado por meio de métodos de divulgação, como oficinas educativas, palestras sobre saúde mental e intervenções com foco nos sintomas de ansiedade, como oficinas de relaxamento e exercícios para aliviar o estresse. Salientase a relevância de intervenções em grupos, que favoreçam a socialização, particularmente úteis entre as estudantes do sexo feminino. ${ }^{19}$

Diante dos riscos e do impacto negativo que o TMC pode causar na qualidade de vida e aprendizado das Rev Enferm Atenção Saúde [Online]. Out/Dez 2021; 10(3):e202136 acadêmicas de enfermagem, bem como seu impacto direto no desempenho acadêmico, faz-se necessário estudos que investiguem a prevalência e os fatores preditivos e as vulnerabilidades acadêmicas, com vistas à implantação de ações estratégicas para prevenção/assistência ao TMC.

Os estudantes de enfermagem são os futuros profissionais, atuantes junto aos pacientes. Sua saúde mental deve ser motivo de preocupação. É fundamental que os enfermeiros tenham bem-estar psicológico para o atendimento competente e seguro aos pacientes. Portanto, salvaguardar o bem-estar psicológico dos estudantes de enfermagem proporciona a garantia da assistência ao paciente segura, eficiente e de alta qualidade. ${ }^{20}$

\section{CONCLUSÕES}

A presente pesquisa evidenciou alta prevalência do TMC entre acadêmicas do curso de Graduação em Enfermagem $(44,7 \%)$. Ainda revelou a associação do uso de álcool e a presença de doença crônica ao TMC.

A partir destas informações foi possível identificar fatores relacionados à saúde mental das acadêmicas, o que pode subsidiar o planejamento de ações específicas contribuindo para a qualidade da sua atuação acadêmica, profissional e pessoal, bem como a aplicação científica, 
tal como a formulação de estudo de intervenção com o mesmo público-alvo.

Limitações do estudo: impossibilidade de inferência de causalidade, devido ao desenho transversal; a realização da pesquisa em uma única universidade e a não utilização do método estatístico multivariado (regressão logística binomial) para a confirmação da análise bivariada.

\section{REFERÊNCIAS}

1. Goldberg DP, Huxley P. Common mental disorders a bio-social model. 4th ed. Londres: Tavistock/Routledge; 1992. $224 \mathrm{p}$.

2. World Health Organization. Depression and other common mental disorders: global health estimates. [Internet] 2th ed. Geneva: WHO; Dec 2017. 24 p. [citado em 23 jan 2019]. Disponível em:

https://apps.who.int/iris/bitstream/hand le/10665/254610/WHO-MSD-MER2017.2eng.pdf? sequence $=1 \&$ isAllowed $=\mathrm{y}$

3. Souza LPS, Barbosa BB, Silva CSO, Souza AG, Ferreira TN, Siqueira LG. Prevalência de transtornos mentais comuns em adultos no contexto da Atenção Primária à Saúde. Rev Port Enferm Saúde Mental [Internet]. 2017 [citado em 13 fev 2019]; (18):59-66. Disponível em: https://scielo.pt/pdf/rpesm/n18/n18a09. pdf

4. Nunes MA, Pinheiro AP, Bessel M, Brunoni AR, Kemp AH, Benseñor IM, et al. Common mental disorders and sociodemographic characteristics: baseline findings of the Brazilian Longitudinal Study of Adult Health (ELSA-Brasil). Rev Bras Psiquiatr. [Internet]. 2016 [citado em 23 jan
2019]; 38(2):91-7. Disponível em: https://www.scielo.br/j/rbp/a/YtCBzHL SGTXQKhFz6znvedd/?format=pdf\&la ng=em

5. Parreira BDM, Goulart BF, Haas VJ, Silva SR, Monteiro JCS, GomesSponholz FA. Common mental disorders and associated factors: a study of women from a rural area. Rev Esc Enferm USP. [Internet]. 2017 [citado em 23 jan 2019]; 51:e03225. Disponível em: https://www.scielo.br/j/reeusp/a/DZ4L VBDqHLDJP43hPQqnzhv/?format=pd f\&lang $=\mathrm{em}$

6. Silva PLBC, Silva BFF, Chagas KKACR, Tortola MBA, Caldeira RLR. Transtorno mental comum entre estudantes de enfermagem e fatores envolvidos. Rev Enferm Cent-Oeste Min. [Internet]. 2019 [citado em 13 fev 2019]; 9:e3191. Disponível em: http://seer.ufsj.edu.br/index.php/recom/ article/view/3191/2165

7. Melo RA, Santos EC, Dias PK. Transtornos mentais comuns em estudantes de administração de uma universidade pública no agreste pernambucano. Journal of Perspectives in Management [Internet]. 2020 [citado em 13 fev 2020]; 4:34-51. Disponível em:

https://periodicos.ufpe.br/revistas/jpm/ article/view/246123/36396

8. Cestari VRF, Barbosa IV, Florêncio RS, Pessoa VLMP, Moreira TMM.

Estresse em estudantes de enfermagem: estudo sobre vulnerabilidades sociodemográficas e acadêmicas. Acta Paul Enferm. [Internet]. 2017 [citado em 13 fev 2019]; 30(2):190-6. Disponível em:

https://www.scielo.br/j/ape/a/4BDZyCJ P6qZ6th7XMtBvhtx/?format=pdf\&lan $\mathrm{g}=\mathrm{pt}$

9. Ferreira CMG, Kluthcovsky ACGC, Cordeiro TMG. Prevalence of common mental disorders and associated factors among medical students: a comparative study. Rev Bras Educ Méd. [Internet]. 
Apr/Jun 2016 [citado em 23 jan 2019]; 40(2):268-77. Disponível em:

https://www.scielo.br/j/rbem/a/jfVpgrY 6MzL5kXsrjD9gH6b/?format=pdf\&lan $\mathrm{g}=\mathrm{pt}$

10. Gonçalves DM, Stein AT, Kapczinski

F. Performance of the self-reporting questionnaire as a psychiatric screening questionnaire: a comparative study with Structured Clinical Interview for DSM-IV-TR. Cad Saúde Pública [Internet]. Feb 2008 [citado em 24 jan 2019]; 24(2):380-90. Disponível em: https://www.scielo.br/j/csp/a/7dgFYgC kbXw9BgwY7dY94Nb/?format=pdf\&l ang $=\mathrm{pt}$

11. Cachoeira DVAC, Santos SCC, Meneganti APS, Negreiros NF, Cardoso L, AlinePreto V. Relation of sociodemographic profile with the risk of illness by mental disorders common among students of the nursing course. Rev Enferm UFPE on line [Internet]. Dec 2016 [citado em 13 fev 2019]; 10(12):4501-8. Disponível em: https://periodicos.ufpe.br/revistas/revist aenfermagem/article/view/11516/1340 1

12. Carleto CT, Moura RCD, Santos VS, Pedrosa LAK. Adaptation to university and common mental disorders in nursing undergraduate student. Rev Eletrônica Enferm. [Internet]. 2018 [citado em 24 jan 2019]; 20a:1-10. Disponível em: https://revistas.ufg.br/fen/article/view/4 3888/25414

13. Costa EFO, Rocha MMV, Santos ATRA, Melo EV, Martins LAN, Andrade TM. Common mental disorders and associated factors among final-year healthcare students. Rev Assoc Med Bras. (1992). [Internet]. Nov/Dec 2014 [citado em 24 jan 2019]; 60(6):525-30. Disponível em: https://www.scielo.br/j/ramb/a/jVvQfty y4cPgRjpfQY5XMGQ/?format=pdf\&1 ang=en

14. Ansolin AGA, Rocha DLB, Santos RP, Dal Pozzo VC. Prevalence of common mental disorder between psychology and nursing students. Arq Ciênc Saúde [Internet]. Out 2015 [citado em 24 jan 2019]; 22(3):42-5. Disponível em: http://www.cienciasdasaude.famerp.br/i ndex.php/racs/article/view/83/103

15. Alfaris E, Irfan F, Qureshi R, Naeem $\mathrm{N}$, Alshomrani A, Ponnamperuma G, et al. Health professions' students have an alarming prevalence of depressive symptoms: exploration of the associated factors. BMC Med Educ. [Internet]. Oct 2016 [citado em 24 jan 2019]; 16(1):279. Disponível em: https://bmcmededuc.biomedcentral.co m/track/pdf/10.1186/s12909-016-0794y.pdf

16. Silva BP, Corradi-Webster CM, Donato ECSG, Hayashida M, Siqueira MM. Common mental disorders, alcohol consumption and tobacco use, among nursing students at a public university in the western Brazilian Amazon. SMAD, Rev Eletrônica Saúde Mental Alcool Drog. [Internet]. May/Aug 2014 [citado em 24 jan 2019]; 10(2):93-100. Disponível em:

http://www.revistas.usp.br/smad/article /view/98724

17. Senicato C, Azevedo RCS, Barros MBA. Transtorno mental comum em mulheres adultas: identificando os segmentos mais vulneráveis. Ciênc Saúde Coletiva [Internet]. ago 2018 [citado em 13 fev 2019]; 23(8):2543-54. Disponível em: https://www.scielo.br/j/csc/a/rSxF9pjY Hk5MwQ3xrvS5zcT/? format=pdf\&lan $\mathrm{g}=\mathrm{pt}$

18. Andrade AS, Antunes NA, Zanoto PA, Tiraboschi GA, Viana PVBA, Curilla RT. Vivências acadêmicas e sofrimento psíquico de estudantes de psicologia. Psicol Ciênc Prof. [Internet]. out/dez 2016 [citado em 13 fev 2019]; 36(4):831-46. Disponível em: https://www.scielo.br/j/pcp/a/RTkfTtD v3sRKHGT7J3zPMZC/?format=pdf\&l ang $=\mathrm{pt}$ 
19. Mao-Sheng Ran, Mendez AJ, Ling-Li L, Bansil B, Reyes N, Cordero G, et al. Predictors of mental health among college students in Guam: implications for counseling. J Couns Dev. [Internet]. Jul 2016 [citado em 24 jan 2019]; 94(3):344-55. Disponível em: https://onlinelibrary.wiley.com/doi/epdf /10.1002/jcad.12091

20. Yi-Jung T, Lo KKH, Ho RCM, Tam WSW. Prevalence of depression among nursing students: a systematic review and meta-analysis. Nurse Educ Today [Internet]. Apr 2018 [citado em $13 \mathrm{fev}$ 2019]; 63:119-29. Disponível em: https://www.sciencedirect.com/science/ article/pii/S0260691718300285?via\%3 Dihub

RECEBIDO: 19/06/2020

APROVADO: 03/09/2021

PUBLICADO: dez/2021 